\section{Serotonin-glutamate receptor complex offers new drug target for psychosis}

Recent advances in antipsychotic therapy have included the development of atypical neuroleptics that function by blocking neurotransmission by the serotonin $(5-\mathrm{HT})$ receptor $2 \mathrm{~A}\left(5-\mathrm{HT}_{2 \mathrm{~A}}\right)$, and agents that function by interacting with the metabotropic glutamate receptor mGluR2 also seem to be effective in this setting. González-Maeso et al. have now found that $5-\mathrm{HT}_{2 \mathrm{~A}}$ and mGluR2 physically and physiologically interact in the brain cortex to form a complex that integrates serotonin and glutamate signaling and might be involved in the development of the psychosis associated with schizophrenia.

The existence of $5-\mathrm{HT}_{2 \mathrm{~A}}-\mathrm{mGluR2}$ complexes was established by several methods, including co-immunoprecipitation experiments, and bioluminescence resonance energy transfer studies. The functional effects of these complexes were demonstrated by experiments conducted with hallucinogens, which induce symptoms that resemble those of psychosis, in mouse somatosensory cortex membranes. In these studies, a glutamate agonist increased the affinity of hallucinogens (5- $\mathrm{HT}_{2 \mathrm{~A}}$ agonists) for the $5-\mathrm{HT}_{2 \mathrm{~A}^{-}}$ binding site. Conversely, a $5-\mathrm{HT}_{2 \mathrm{~A}}$ agonist decreased the affinity of mGluR2 agonists for glutamate receptor binding. In vivo mouse studies demonstrated that hallucinogen-induced head-twitch movements were diminished in the presence of mGluR2 agonists. In addition, postmortem studies demonstrated increased levels of $5-\mathrm{HT}_{2 \mathrm{~A}}$ and decreased levels of mGluR2 in the brains of patients with untreated schizophrenia.

In light of these data, the authors identify the $5-\mathrm{HT}_{2 \mathrm{~A}}-\mathrm{mGluR2}$ complex as a mediator between the serotonin and glutamate neurotransmitter systems and as a potential drug target for the treatment of psychosis in schizophrenia.

Original article González-Maeso J et al. (2008)

Identification of a serotonin/glutamate receptor complex implicated in psychosis. Nature 452: 93-97

\section{Transmission of multiple sclerosis might be mediated through maternal relatives}

Previous studies have produced conflicting evidence regarding the possible maternal or paternal transmission of multiple sclerosis (MS).
The results of a study conducted in a genetically isolated population in the The Netherlands with genealogical information going back to the $18^{\text {th }}$ century support a maternal parent-of-origin effect in MS.

The study by Hoppenbrouwers et al. included 24 individuals with MS $(79 \%$ women; mean age at onset $29 \pm 9$ years; $83 \%$ with relapse-onset MS) none of whom had parents or grandparents with MS. On the basis of genealogical data spanning 23 generations, which were obtained from the Genetic Research in Isolated Populations program, it was determined that mothers of individuals with MS were almost four times as closely related to each other than fathers were (mean kinship 0.0031 vs $0.0008 ; P<0.001$ ). Between two individuals with MS, the shortest connection to a common ancestor was more often through a mother than a father-among 814 of these shortest connections, 333 (40.9\%) were between mothers, 98 (12.0\%) were between fathers and $383(47.1 \%)$ were between a father and mother. Without a parent-of-origin effect, the expected distributions would be $25 \%, 25 \%$ and $50 \%$, respectively. In addition, the researchers investigated parent-of-origin effects for Parkinson's disease, late-onset Alzheimer's disease and diabetes mellitus. No such effect was seen for any of these diseases, indicating that the effect might be specific to MS.

The authors suggest that the maternal parent-of-origin effect in MS might be due to a gene-environment effect that takes place in utero.

Original article Hoppenbrouwers IA et al. (2008) Maternal transmission of multiple sclerosis in a Dutch population. Arch Neurol 65: 345-348

\section{SSRIs show efficacy over placebo only in the most severely depressed patients}

Selective serotonin reuptake inhibitors (SSRIs) have been approved for the treatment of depression but have failed to show clear clinical efficacy versus placebo when examined in meta-analyses. Kirsch et al. have proposed that analysis of patients as a single group might obscure a treatment effect in those who are most likely to benefit from these agents, such as individuals with extremely severe depression. 